\title{
ZUR SOZIALGESCHICHTE DER ARMENFÜRSORGE IN DEN STÄD- TEN DES SÜDLICHEN OSTSEERAUMS (13.-16. JAHRHUNDERT)
}

\author{
EIN FORSCHUNGSPROJEKT \\ von Sven Rabeler
}

Am 24. Juli 1473 ließ Hans Berskamp († ca. 1486 [?]), Bürger zu Lübeck, ein Testament aufsetzen.' Darin traf er u. a. Verfügungen über den elenden hagen - also den Armengang - in der Lübecker Schlumacherstraße. Fünfzehn Jahre zuvor - 1458 - hatte Berskamp gemeinsam mit Hermann Evinghusen $(\dagger 1483)$ das betreffende Grundstück erworben. ${ }^{2}$ Beide hatten dort ein neues Vorderhaus mit einem rückwärts anschließenden Gang errichtet; letzterer umfasste nach Auskunft des angeführten Testamentes von 1473 dreizehn Buden. Berskamp legte fest, dass in zwölf der Buden je zwei Personen kostenlose Unterkunft finden sollten. Die dreizehnte Behausung war zur Wohnung des „Vogtes" bestimmt, der dem gesamten Hof vorzustehen hatte und ebenfalls kostenloses Logis erhielt. Das Vorderhaus war in vier Wohnungen geteilt, für die - im Gegensatz zu den Gangbuden - Miete eingefordert wurde. Diese Einkünfte - so legte Berskamp fest sollten der baulichen Unterhaltung, darüber hinaus den im Gang lebenden armen luden zur Versorgung mit Kohlen dienen. Als Hermann Evinghusen vier Jahre später - 1477 - ebenfalls seinen Letzten Willen festhalten ließ, bestätigte er die gemeinsam mit Hans Berskamp gefassten Beschlüsse hinsichtlich des Armenganges und der dortigen Unterbringung von 25 gude[n] lude[n]. ${ }^{3}$ Zusätzlich setzte er jährlich 5 Mark für den weiteren Zukauf von Kohlen aus.

Die Stiftung von Armenhäusern war in einer spätmittelalterlichen Stadt kein ungewöhnlicher Vorgang. ${ }^{4}$ Im Fall Lübecks lassen sich für rund zwei

' Archiv der Hansestadt Lübeck (im Folgenden: AHL), Test. 1473 Juli 24. - Der Text gibt den am 7. Juni 2006 auf der Jahresversammlung des Hansischen Geschichtsvereins in Osnabrück gehaltenen Vortrag in leicht überarbeiteter Form wieder, die Fußnoten beschränken sich auf unbedingt notwendige Nachweise und wenige exemplarische Literaturangaben.

${ }^{2}$ AHL, Handschrift $900 \mathrm{~b}$ [Schrödersche Oberstadtbuch-Regesten, Johannis-Quartier], S. 241; Johannis-Quartier 207-211 = Schlumacherstraße 15-23.

${ }^{3}$ AHL, Test. 1477 Sept. 23.

${ }^{+}$Zu Armenhäusern s. allgemein anhand englischer Beispiele Frank REXROTH, Armen- 
Dutzend derartiger Häuser und Gänge nähere Angaben machen. Sie bildeten zusammen mit Hospitälern anderer Art, wie zum Beispiel dem Heilig-Geist-Hospital und den vor den Toren der Stadt gelegenen Siechenhäusern, sowie mit einer Vielzahl weiterer karitativer Stiftungen, die der Speisung und Bekleidung von Armen und zahlreichen anderen Zwecken dienten, einen Teil der komplexen, aus heutiger Sicht verwirrend vielfältigen Armenfürsorge in Lübeck, die in manchen Bereichen durch Kontinuitäten über das Mittelalter hinaus gekennzeichnet ist: Berskamps und Evinghusens Armengang existierte über Jahrhunderte hin, wobei in der Neuzeit die Bezeichnung als ,von Dorne-Gang“ üblich wurde. ${ }^{5}$ Erhalten ist bis heute das im 15. Jahrhundert errichtete Vorderhaus. ${ }^{6}$

Hans Berskamp und Hermann Evinghusen traten - dies ist für das Folgende ein bedeutsamer Punkt - gemeinsam als Stifter auf. Die soziale Beziehung beider Personen, die ein solches Vorhaben motivieren konnte, war anscheinend nicht verwandtschaftlicher Art. Möglicherweise bestanden Geschäftsverbindungen. Exemplarisch lässt sich aber festhalten, dass die karitative Stiftung hier bereits von ihren Voraussetzungen her als soziale Interaktion erscheint, dass dahinter ein soziales Beziehungsgefüge stand.

I.

Das Forschungsprojekt, das im Folgenden mit einigen skizzenhaften Überlegungen zur Konzeption, ergänzt durch wenige illustrierende Beispiele, vorgestellt wird, beschäftigt sich mit Armenfürsorge und karitativen Stiftungen als Formen sozialer Interaktion. ${ }^{7}$ Zwar war und ist die städtische Armenfürsorge des Mittelalters Gegenstand sehr intensiver Forschungen, auch wenn die kaum mehr überschaubare Fülle an Publikationen über Armut, Hospitäler, karitatives Stiftungswesen und alle Arten der Fürsorge eine gewisse Vernachlässigung der Städte des südlichen Ostseeraums zeigt. Doch lässt der komplexe Forschungsstand, den darzustellen an

häuser - eine neue Institution der sozialen Fürsorge im späten Mittclalter, in: Funktions- und Strukturwandel (wie Anm. 8), S. 1-14.

${ }^{5}$ Zum Übergang der Verwaltung auf die Familie von Dorne s. Verzeichniß der PrivatWohltätigkeits-Anstalten im Lübeckischen Freistaate, Lübeck 1901, S. $30 \mathrm{f}$.

${ }^{6}$ Kunst-Topographie Schleswig-Holstein (Die Kunstdenkmäler des Landes SchleswigHolstein), Neumünster 1969, S. 107.

${ }^{7}$ Die Arbeit, die am Historischen Seminar der Christian-Albrechts-Universität zu Kiel (Lehrstuhl Prof. Dr. Gerhard Fouquet) durchgeführt wird, wird seit 2005 unter dem Titel „Armenfürsorge im sozialen Interaktionsraum Stadt: Karitative Stiftungen im südlichen Ostseeraum des Mittelalters (13.-16. Jahrhundert)“ durch die Deutsche Forschungsgemeinschaft (DFG) finanziert. 
dieser Stelle unmöglich ist, ${ }^{8}$ darüber hinaus grundsätzliche Defizite erkennen. In holzschnittartiger Manier, unter Vernachlässigung zahlreicher Einzelheiten sei hier nur auf drei Probleme hingewiesen, die erstens den interpretatorischen Kontext, zweitens die inhaltliche Ausrichtung und drittens den methodischen Zugang betreffen.

1. Die mittelalterliche Caritas erscheint - wenn sie nicht als vornehmlich religiöses Phänomen gedeutet wird - nicht selten quasi als Vorgeschichte der neuzeitlichen Armenfürsorge. Ob die ältere Forschung versuchte, die Vorläufer moderner Wohlfahrtspolitik in den Städten des Mittelalters zu finden," ob die jüngere Forschung danach strebte, das für die frühe Neuzeit entwickelte und nicht unumstrittene Konzept der ,Sozialdisziplinierung " auf das 14. und 15. Jahrhundert zu übertragen: ${ }^{10}$ das Mittelalter wird retrospektiv wahrgenommen. Aber auch wenn nach Kontinuitäten, Anknüpfungspunkten, Ursprüngen gefragt wird, so erscheint das Mittelalter doch vornehmlich als Gegenbild moderner sozialpolitischer Vorstellungen: unvollkommen, disfunktional, irrational. Erst in der Reformationszeit - so eine bis heute gängige Forschungsmeinung - habe man versucht, diesem karitativen Chaos ein Ende zu bereiten, vorerst allerdings

${ }^{8}$ Statt dessen sei hier allein auf einige neuere Sammelbände hingewiesen, die aktuelle Themen und Tendenzen der Forschung widerspicgeln: Städtisches Gesundheits- und Fürsorgewesen vor 1800, hg. von Peter JOHANEK (Städteforschung A 50), Köln 2000; Von der Barmherzigkeit zur Sozialversicherung. Umbrüche und Kontinuitäten vom Spätmittelalter bis zum 20. Jahrhundert, hg. von Hans-Jörg GILOMEN, Sébastien GuEX und Brigitte STUDER (Schweizerische Gesellschaft für Wirtschafts- und Sozialgeschichte 18), Zürich 2002; Armut im Mittelalter, hg. von Otto Gerhard OEXLE (Vorträge und Forschungen 58), Ostfildern 2004; Inklusion/Exklusion. Studien zu Fremdheit und Armut von der Antike bis zur Gegenwart, hg. von Andreas Gestrich und Lutz RAPHAEL, Frankfurt am Main 2004; Das Hospital am Beginn der Neuzeit. Soziale Reform in Hessen im Spiegel europäischer Kulturgeschichte, hg. von Arnd FrIEDRICH, Fritz HEINRICH und Christine VANJA (Historische Schriftenreihe des Landeswohlfahrtsverbandes Hessen, Quellen und Studien 11), Petersberg 2004: Funktionsund Strukturwandel spätmittelalterlicher Hospitäler im europäischen Verglejch, hg. von Michael MATHEUS (Geschichtliche Landeskunde 56), Stuttgart 2005; Norm und Praxis der Armenfürsorge in Spätmittelalter und früher Neuzeit, hg. von Sebastian SCHMIDT und Jens ASPELMEIER (VSWG, Beihefte 189), Stuttgart 2006.

${ }^{9}$ Walter MörING. Die Wohlfahrtspolitik des Hamburger Rates im Mittelalter (Abhandlungen zur mittleren und neueren Geschichte 45), Berlin 1913: Robert AHRENS, Die Wohlfahrtspolitik des Rostocker Rats bis zum Ende des 15. Jahrhunderts, Diss. phil. Rostock 1927; Hermann LaGEMANN. Polizeiwesen und Wohlfahrtspflege in Lübeck von den Anfängen bis zum Ende des 16. Jahrhunderts. Schönberg (Meckl.) 1916.

${ }^{10} \mathrm{Zu}$ den unterschiedlichen Positionen der Frühneuzeitforschung vgl. Martin Dinges, Frühneuzeitliche Armenfürsorge als Sozialdisziplinierung? Probleme mit einem Konzept, in: Geschichte und Gesellschaft 17, 1991, S. 5-29, und die Entgegnung von Robert JüTTE, .Disziplin zu predigen ist eine Sache, sich ihr zu unterwerfen eine andere" (Cervantes). Prolegomena zu einer Sozialgeschichte der Armenfürsorge diesseits und jenseits des Fortschritts, in: Geschichte und Gesellschaft 17, 1991, S. 92-101, zur Übertragung auf das Spätmittelalter z. B. Werner BUCHHOLZ. Anfänge der Sozialdisziplinierung im Mittelalter. Die Reichsstadt Nürnberg als Beispiel, in: ZHF 18, 1991, S. 129-147. 
ohne nachhaltigen Erfolg. Nun lassen sich in der mittelalterlichen Armenfürsorge zweifellos nicht ohne weiteres Konzepte moderner Rationalität erkennen. Das bedeutet aber keineswegs, dass die vielgestaltige Entwicklung karitativer Systeme, wie ich sie im Fall Lübecks kurz angedeutet habe, keinerlei Ratio gefolgt wäre.

2. Die Geschichte der mittelalterlichen Armenfürsorge ist bis heute nicht ausschließlich, aber überwiegend die Geschichte ihrer Institutionen, d. h. vor allem der Hospitäler. Und Hospitalgeschichte wiederum wird vorrangig verstanden als Geschichte des Hospitalrechts und der Hospitalökonomie." Diese deutliche Bevorzugung eines institutionsgeschichtlichen Zuganges führt dazu, dass die Einbindung in die städtische Sozialgeschichte insgesamt unzureichend erscheint. Damit hängt auch die Frage nach der spezifischen Funktion der mittelalterlichen Armenfürsorge zusammen, zu der das Stichwort ,Memoria', also die Pflege des Totengedächtnisses, ${ }^{12}$ nur eine - wenngleich wichtige - Teilantwort liefert.

3. Vorherrschend ist die Tendenz zur isolierten Betrachtung einzelner karitativer Handlungsfelder. Thematisch einschlägige Arbeiten beschäftigen sich in der Regel entweder mit Hospitälern ${ }^{13}$ oder mit Testamenten ${ }^{14}$ oder mit der städtischen Normengebung. ${ }^{15}$ Selten werden zudem inner-

" Grundlegend aus rechtsgeschichtlicher Sicht ist bis heute Siegfried REICKE, Das deutsche Spital und sein Recht im Mittelalter, 2 Teile (Kirchenrechtliche Abhandlungen, 111-114), Stuttgart 1932.

${ }^{12}$ Ralf LUSIARDI, Stiftung und städtische Gesellschaft. Religiöse und soziale Aspekte des Stiftungsverhaltens im spätmittelalterlichen Stralsund (Stiftungsgeschichten 2), Berlin 2000; Benjamin SCHEller, Memoria an der Zeitenwende. Die Stiftungen Jakob Fuggers des Reichen vor und während der Reformation (ca. 1505-1555) (Stiftungsgeschichten 3), Berlin 2004.

${ }^{13}$ Erich ZECHLIN, Lüneburgs Hospitäler im Mittelalter (Forschungen zur Geschichte Niedersachsens 1/6), Hannover 1907; Ernst-Adolf MEINERT, Die Hospitäler Holsteins im Mittelalter. Ein Beitrag zur mittelalterlichen Stadtgeschichte (Quellen und Forschungen zur Geschichte Schleswig-Holsteins 107), Neumünster 1997 [Druck der masch. Diss. Kiel 1949]; Rudolf KLEIMINGER, Das Heiligengeisthospital von Wismar in sieben Jahrhunderten. Ein Beitrag zur Wirtschaftsgeschichte der Stadt, ihrer Höfe und Dörfer (Abhandlungen zur Handels- und Sozialgeschichte 4), Weimar 1962; Wolfgang BERGER, Das St.-Georgs-Hospital zu Hamburg. Die Wirtschaftsführung eines mittelalterlichen Großhaushalts (Beiträge zur Geschichte Hamburgs 8), Hamburg 1972; Harald Schulz, Studien zur Wirtschafts- und Sozialgeschichte des Heilig-Geist-Hospitals zu Lübeck, Diss. phil. Göttingen 1993; Frank HATJE, „Golt zu Ehren, der Armut zum Besten“. Hospital zum Heiligen Geist und Marien-Magdalenen-Kloster in der Geschichte Hamburgs vom Mittelalter bis in die Gegenwart, Hamburg 2002.

${ }^{14}$ Carsten Selch JenSEN, Fromme gaver i senmiddelalderlige lybske testamenter, Diss. Odense 1997 (masch.); Birgit NOODT, Religion und Familie in der Hansestadt Lübeck anhand der Bürgertestamente des 14. Jahrhunderts (Veröffentlichungen zur Geschichte der Hansestadt Lübeck B 33), Lübeck 2000.

${ }^{15}$ Willi RÜGER, Mittelalterliches Almosenwesen. Die Almosenordnungen der Reichsstadt Nürnberg (Nürnberger Beiträge zu den Wirtschafts- und Sozialwissenschaften 31), Nürnberg 1932; Robert JÜTTE, Obrigkeitliche Armenfürsorge in deutschen Reichsstädten der frühen 
städtische und zwischenstädtische Vergleiche konsequent in die Untersuchung einbezogen.

Vor dem Hintergrund dieser Defizite verfolgt das vorgestellte Projekt einen Ansatz, der sich in neuere Zugänge der Forschung zu diesem alten und immer wieder jungen, weil aktuellen Thema einordnet: eine vergleichende Sozialgeschichte der ,Armenfürsorge" in den Städten einer Region, nämlich des südlichen Ostseeraums, wobei Lübeck, Hamburg, Lüneburg, Wismar und Kiel Schwerpunkte bilden, aber auch andere holsteinische, schleswigsche, nordostniedersächsische, mecklenburgische und pommersche Städte einbezogen werden. Inhaltlich geht es nicht so sehr um Armut an sich, etwa um Lebensformen von Armen, Hospitalbewohnern und Bettlern. ${ }^{16}$,Armenfürsorge ${ }^{6}$ und karitatives Handeln werden vielmehr als Teil von Interaktions- und Kommunikationsprozessen innerhalb des sozialen, ökonomischen und politischen ,Systems “ der Städte des südlichen Ostseeraums aufgefasst. Ziel ist die Analyse von Formen karitativen Handelns im Kontext städtischer Sozialbeziehungen: Wie reagierten Personen und Gruppen innerhalb des sozialen Interaktionsraums der mittelalterlichen Stadt auf ,Armut ${ }^{\circ}$ in ihren unterschiedlichen Ausprägungen? Gerade im Bereich der ,Armenfürsorge" kristallisieren sich in besonderer Weise soziale Netzwerke und Beziehungsformen heraus, die z. B. familiale und klientelistische Strukturen prägten, aber auch für die gesamte ,Verfasstheit ' von Städten mit ihren genossenschaftlichen und herrschaftlichen Elementen bestimmend waren. Wie gingen Familien und korporative Gruppen, kirchliche Institutionen, Rat, Gemeinde und Stadtherr mit karitativen Stiftungen um? Wie gestalteten sich ihre jeweiligen Interessen auf dem Handlungsfeld ,Armenfürsorge', wie agierten sie in diesem Zusammenhang neben-, mit- oder gegeneinander? Welche Beziehungen bestanden zwischen den Trägern karitativer Stiftungen? Insgesamt soll das Projekt Aufschlüsse über soziale, politische und kulturelle Praktiken, Mechanismen und Verhaltensformen geben.

Neuzeit. Städtisches Armenwesen in Frankfurt am Main und Köln (Kölner historische Abhandlungen 31), Köln 1984; Ernst SCHUBERT, Der „starke Bettler“. Das erste Opfer sozialer Typisierung um 1500, in: ZfG 48, 2000, S. 869-893.

${ }^{16}$ Valentin GROEBNER, Ökonomie ohne Haus. Zum Wirtschaften armer Leute in Nürnberg am Ende des 15. Jahrhunderts (Veröffentlichungen des Max-Planck-Instituts für Geschichte 108), Göttingen 1993; Gerhard FoUQUET, Familie, Haus und Armut in spätmittelalterlichen Städten - Das Beispiel des Augsburger Ehepaares Elisabeth Störkler und Burkard Zink, in: Inklusion/Exklusion (wie Anm. 8). S. 283-307; Helmut BRÄUER. Der Leipziger Rat und die Bettler. Quellen und Analysen zu Bettlern und Bettelwesen in der Messestadt bis ins 18. Jahrhundert, Leipzig 1997; Ernst SCHUBERT, Erscheinungsformen der Armut in der spätmittelalterlichen deutschen Stadt, in: Die Stadt als Kommunikationsraum. Beiträge zur Stadtgeschichte vom Mittelalter bis ins 20. Jahrhundert. Festschrift für Karl Czok zum 75. Geburtstag, hg. von Helmut BRÄUER und Elke SCHLENKRICH, Leipzig 2001, S. 659-697. 
Methodisch zurückzugreifen ist dabei auf personengeschichtliche Verfahren, so dass nicht nur zu Stiftungen, sondern vor allem auch zu Stiftern und Vorstehern Daten gesammelt und Kataloge angelegt werden. Für Lübeck beispielsweise umfassen diese Kataloge momentan gut 40 Stiftungen und rund 240 Personen. Für die weitere Auswertung wichtig sind die Verflechtungsanalyse ${ }^{17}$ und das Konzept der Soziabilitätsforschung. ${ }^{18}$ Letzteres meint die Untersuchung konkreter Handlungen innerhalb sozialer Beziehungs- und Gruppengeflechte einschließlich der Selbstdeutung der beteiligten Akteure. Diese Mikroebene ist in Analyse und Darstellung mit der sozialen, ökonomischen und politischen Entwicklung der behandelten Städte im Ganzen zu verklammern.

$\mathrm{Zu}$ den wichtigen methodischen Elementen des Projektes gehört außerdem der Vergleich zwischen den Städten des südlichen Ostseeraums. Diese weisen zwar eine Reihe rechtlicher, politischer und sozioökonomischer Gemeinsamkeiten auf, zeigen aber auch Unterschiede, z. B. hinsichtlich Größe, wirtschaftlicher Ausrichtung, Zahl und Art der kirchlichen Institutionen, Stellung zum Stadtherrn. Das vergleichende Vorgehen kann den Einfluss derartiger struktureller Abweichungen auf die "Armenfürsorge" erkennbar machen, die differenziertere Betrachtung der Ergebnisse ermöglichen, eine eventuelle Verallgemeinerung im regionalen Kontext absichern bzw. präzisieren und die stadtübergreifende Verflechtung karitativen Handelns verdeutlichen, wie sie wenigstens in einzelnen Fällen festzustellen ist.

Bedingt durch den Stadtwerdungsprozess in der behandelten Region und das Einsetzen relevanter Quellen in größerer Zahl beginnt der Untersuchungszeitraum im Wesentlichen mit dem 13. Jahrhundert. Er endet unter Einschluss der Reformationszeit ungefähr Mitte des 16. Jahrhunderts. Die Arbeit basiert zu großen Teilen auf ungedruckten Quellen, u. a. auf der systematischen Durchsicht der einschlägigen Archive in Lübeck, Hamburg, Lüneburg, Wismar und Kiel.

Zur näheren inhaltlichen Bestimmung des Projektes soll auf drei Kriterien kurz eingegangen werden: auf den Armutsbegriff als Kennzeichnung des Objekts karitativen Handelns (II), auf karitative Stiftungen als Instrument (III) und auf die Stadt als, sozialen Ort (IV).

\footnotetext{
${ }^{17}$ Wolfgang REINHARD, Freunde und Kreaturen. „Verflechtung“ als Konzept zur Erforschung historischer Führungsgruppen. Römische Oligarchie um 1600 (Schriften der Philosophischen Fachbereiche der Universität Augsburg 14), München 1979.

${ }^{18}$ Simon TEuSCHER, Bekannte - Klienten - Verwandte. Soziabilität und Politik in der Stadt Bern um 1.500 (Norm und Struktur 9), Köln 1998.
} 
II.

In ihren Bemühungen um die Definition von "Armut" in der Vormoderne ist die Forschung zu durchaus unterschiedlichen Resultaten gelangt. ${ }^{19} \mathrm{Im}$ Rahmen des Projektes wird auf die Bildung eines ,objektiven" Armutsbegriffes verzichtet, vielmehr soll von einem ,diskursiven' Armutsbegriff ausgegangen werden. Bereits die Zuschreibung von ,Armut", wie sie uns in den Quellen entgegentritt, wird als Form sozialer Interaktion und Kommunikation verstanden. Die Analyse kann sich in der behandelten Region zwar kaum auf Zeugnisse eines expliziten Armutsdiskurses - etwa in Form theoretischer Schriften oder Traktate ${ }^{20}$ - stützen, doch Testamente, Rechnungsbücher, Hospitalordnungen und andere Texte zeugen von einem impliziten Diskurs. Dies ermöglicht heuristisch die Einbeziehung des Quellenbegriffes "Armut ${ }^{6}$ in seinen verschiedenen Varianten, analytisch die differenzierte Beschreibung und Kontextualisierung der Quellen, interpretatorisch den Verzicht auf eine vorab definierte und damit den Gegenstand bereits isolierende Kategorienbildung. Praktisch bedeutet dies, dass ,Armenfürsorge" im Sinne des Projektes nicht nur Reaktionen auf unmittelbare materielle Bedürftigkeit und existenzielle Not einschließt, sondern z. B. auch Stipendienstiftungen für ,arme' Schüler oder die Bereitstellung der Aussteuer für ,arme' Mädchen. Die Diskursanalyse dient so der Gewinnung eines begrifflichen Arbeitsinstrumentes, das auf sozialen Beziehungen basiert, aber auch der Rückbindung an ökonomische Faktoren bedarf.

Die Bedeutungsvarianten des Wortfeldes ,arm" und seiner lateinischen Entsprechung pauper lassen sich beispielsweise an den Hamburger Testamenten der zweiten Hälfte des 14. Jahrhunderts ausschnitthaft verdeutlichen. ,Arme " können in diesen Texten naturgemäß in einem christologischen oder memorialen Sinnzusammenhang vorkommen, etwa in der

\footnotetext{
${ }^{19}$ Thomas FiSCHER, Städtische Armut und Armenfürsorge im 15. und 16. Jahrhundert. Sozialgeschichtliche Untersuchungen am Beispiel der Städte Basel, Freiburg i. Br. und Straßburg (Göttinger Beitrïge zur Wirtschafts- und Sozialgeschichte 4), Göttingen 1979. S. 17-139; Wolfgang von HIPPEL, Armut, Unterschichten, Randgruppen in der frühen Neuzeit (Enzyklopädie deutscher Geschichte 34), München 1995, S. 3-7; SCHUBERT, Erscheinungsformen (wie Anm. 16). Zur begrifflichen Differenzierung in spätmittelalterlichen Quellen s. Ernst SCHUBERT, ..Hausarme Leute“, „starke Bettler": Einschränkungen und Umformungen des Almosengedankens um 1400 und um 1500, in: Armut im Mittelalter (wie Anm. 8), S. 283-347.

${ }^{20} \mathrm{Zu}$ einem prominenten Beispiel des späten 15. Jh.s s. Rita VOLTMER, Zwischen polittheologischen Konzepten, obrigkeitlichen Normsetzungen und städtischem Alltag: Die Vorschläge des Straßburger Münsterpredigers Johannes Geiler von Kaysersberg zur Reform des städtischen Armenwesens, in: Norm und Praxis der Armenfürsorge (wie Anm. 8), S. 91-135.
} 
Formulierung pauperes Cristi $^{21}$ oder in Verbindung mit dem Ausdruck pro salute anime mee. ${ }^{22}$ Auch gänzlich unreflektiert erscheinen arme lude als Gabenempfänger, ${ }^{23}$ und erwartungsgemäß werden Hospitalbewohner häufig mit dem Adjektiv ,arm“ bedacht. ${ }^{24}$ Vorerst noch isoliert taucht in einem Testament von 1357 als nähere Bestimmung der Begriff „Hausarme“ auf, der seit dem 15. Jahrhundert zentrale Bedeutung gewinnt. ${ }^{25} \mathrm{Ab}$ dem späten 14. Jahrhundert werden in den Hamburger Testamenten dann vermehrt spezifische Kriterien verwendet, um den Kreis der Empfänger bestimmter Leistungen zu definieren. So zeigt das Testament, das Heine Wilde 1388 aufsetzen ließ, recht genau die Vorgaben des Ausstellers bei der Vergabe karitativer Zuwendungen: 12 Mark waren jährlich an arme lude in der Petrikirche mit Wissen der dortigen Kirchgeschworenen zu verteilen. Arme juncvrowen, den ys noet ys und die aus der Familie Krowel und damit aus der väterlichen Verwandtschaft des Testators stammen mussten, sollten einmalig 30 Mark Pfennige erhalten. Und schließlich hatten die Exekutoren den Rest des Vermögens, der nach Abrechnung aller testamentarischen Legate übrigbleiben würde, armen juncvrowen zuzuwenden, de myne vrunt syn - die also ebenfalls der Verwandtschaft des Heine Wilde angehörten -, wohl in Ermangelung entsprechender Anwärterinnen auch armen presteren und armen luden. ${ }^{26}$ Das Beispiel zeigt, wie oftmals kleinere soziale und topographische Einheiten - in diesem Fall das Kirchspiel St. Petri - sowie verwandtschaftliche Beziehungen zu Bestimmungsfaktoren für die Auswahl der Empfänger karitativer Leistungen wurden. Die Aneinanderreihung von armen Verwandten, armen Priestern und (anderen) armen Leuten verdeutlicht die differenzierte und zugleich doch zusammengehörige, im zeitgenössischen Verständnis offenbar parallele Anwendung des Begriffes ,arm“.

III.

Für eine Sozialgeschichte der mittelalterlichen ,Armenfürsorge' sind karitative Stiftungen von besonderer Relevanz. ${ }^{27}$ Stiftungen zeichneten sich

\footnotetext{
${ }^{21}$ Hans Dieter LoOSE (Bearb.), Hamburger Testamente 1351 bis 1400 (Veröffentlichung aus dem Staatsarchiv der Freien und Hansestadt Hamburg 11), Hamburg 1970, Nr. 4 (S. 3 und 7).

${ }^{22}$ LOOSE, Testamente (wie Anm. 21), Nr. 3, 12 und 29.

${ }^{2.3}$ LOOSE, Testamente (wie Anm. 21), Nr. 46, 52, 84 (S. 90), 86, 100, 102, 106, 118, 119 und 129 .

${ }^{24}$ Loose, Testamente (wie Anm. 21), Nr. 58, 65, 97 (S. 104), 102, 106, 115 und 127.

${ }^{25}$ LOOSE, Testamente (wie Anm. 21), Nr. 7 (S. 12): [...] pauperibus husarmen dictis [...]. - Zum Begriff „Hausarme“ s. SCHUBERT, „Hausarme Leute“ (wie Anm. 19).

${ }^{26}$ LOOSE, Testamente (wie Anm. 21), Nr. 79 (S. 84).
} 
in der sozialen Praxis allgemein durch eine hohe Komplexität aus, da sie auf Ewigkeit angelegt waren. Im einfachsten Fall setzte der Stifter mehrere Vorsteher ein, die sich später mittels Kooptation selbst ergänzten. So bestimmten zum Beispiel auch die eingangs erwähnten Lübecker Bürger Hans Berskamp und Hermann Evinghusen in ihren Testamenten von 1473 bzw. 1477 namentlich genannte Nachlassexekutoren, mit denen wir Teile der - auch hier partiell gemeinsamen - sozialen Verflechtung greifen. Gerade im Fall karitativer Stiftungen konnten aber die Verwaltungs- und Kontrollmechanismen und die Einbindung in die sozialen, politischen und rechtlichen Strukturen der Stadt weit komplizierter gestaltet sein. Dabei setzten die Beteiligten - insbesondere die Vorsteher - keineswegs allein den Stifterwillen um, war ihr Handeln doch durch eigene Interessen und Beziehungen geprägt. ${ }^{28}$ Weiterhin wiesen karitative Stiftungen zwar Gemeinsamkeiten mit anderen Stiftungsformen auf - z. B. im Hinblick auf die damit verbundenen memorialen Aspekte -, zeigten aber insofern grundsätzliche Unterschiede, als sie weitergehende pragmatische Funktionen erfüllten, die mit den individuellen Intentionen des jeweiligen Stifters nicht deckungsgleich zu sein brauchten. Daraus folgte eine besondere Personenkonstellation, zu der nicht nur Stifter und Vorsteher gehörten, sondern auch die ,Armen“ als ,Objekte“ ihres Handelns. Aus dem Charakter karitativer Stiftungen resultierten weiterhin spezifische Regelungsbedürfnisse, z. B. die Auswahl der Leistungsempfänger, die nach unterschiedlichen Kriterien erfolgen konnte, und die Ordnung des Zusammenlebens der Bewohner von Hospitälern. Hans Berskamp sprach 1473 anders als viele andere Stifter - von den Bewohnern des Ganges in der Lübecker Schlumacherstraße undifferenziert als armen luden, aber auch er gab beispielsweise durch die Einsetzung eines Vogtes Richtlinien für das Zusammenleben der Gemeinschaft vor. Für andere Hospitäler kennen wir bereits seit dem 13. Jahrhundert Satzungstexte, im Fall des von Hans Berskamp und Hermann Evinghusen gestifteten Armenganges stammt die erste erhaltene Ordnung aus dem Jahr $1579 .{ }^{29}$ Erlassen hat sie der damalige Vorsteher Lukas Lange.

Die Handlungsmöglichkeiten von Vorstehern karitativer Stiftungen, die für das Projekt von großem Interesse sind, lassen sich zum Beispiel

${ }^{27}$ Michael BORGOLTE, Die Stiftungen des Mittelalters in rechts- und sozialhistorischer Sicht, in: Zs. der Savigny-Stiftung für Rechtsgesch. 105, Kan. Abt. 74, 1988, S. 71-94: Stiftungen und Stiftungswirklichkeiten. Vom Mittelalter bis zur Gegenwart, hg. von Michael BORGOLTE (Stiftungsgeschichten 1), Berlin 2000.

${ }^{28}$ Sven RABELER, Karitatives Handeln, Stiftungswirklichkeiten und Personenbeziehungen - Überlegungen zu einer Sozialgeschichte der Armenfürsorge im mittelalterlichen Lübeck, in: ZVLGA 85, 2005, S. 11-24.

29 AHL, Kleinere Stiftungen, von Dorne Stift, Nr. 5. 
anhand des Lüneburgers Hinrich Lange näher aufzeigen, was hier wiederum nur mit einigen knappen Hinweisen angedeutet werden kann. Seit 1410 war Hinrich Lange, der erst 1431 in den Lüneburger Rat gewählt wurde und ab 1448 als Bürgermeister amtierte, ${ }^{30}$ Helfer und de facto Vertreter des Ratsherrn Johann Rese als Vorsteher des Nikolaihofes, eines unweit von Lüneburg in Bardowick gelegenen Aussätzigenhospitals. Als Rese 1431 starb, folgte ihm Lange auch offiziell im Amt des Vorstehers nach. Bis zu seinem Tod im Jahre 1467 übte er diese Funktion aus. Zeugnis davon legt ein umfangreiches Rechnungsbuch ab, das Lange von 1410 bis 1467 eigenhändig führte und das uns über viele Details seiner Tätigkeit informiert. ${ }^{31}$ In einer anderen Handschrift, die z. B. einen Memorienkalender des Nikolaihofes enthält, findet sich zu Hinrich Lange ein Eintrag, der die Wohltaten des verstorbenen Vorstehers zugunsten ,seines' Hospitals über vier Seiten hin aufzählt: Ankäufe - insbesondere von Salzgütern -, Baumaßnahmen, wozu vor allem der Neubau der Kirche im Jahre 1435 gehörte, deren Weihe Hinrich Lange - wir erfahren es aus seinem Rechnungsbuch $^{32}$ - auch angemessen in Szene zu setzen wusste, zusätzliche Geldverteilungen an die Armen und vieles mehr - kurzum alles, was Lange nach Ansicht des anonymen Autors zum Besten der Armen geleistet hatte (unde hefft den armen luden buwet unde tokofft unde vorbeterd ere gudere, alze hirna screven steyt [...]). ${ }^{33}$ Auch wenn der Nikolaihof der Aufsicht des Lüneburger Rates unterstand, mit einem gängigen Begriff der Forschung also „kommunalisiert“"war - in der Praxis erwies sich das individuelle Handeln des Vorstehers als entscheidend und damit als erinnerungswürdig. Auf etwas andere Weise beleuchtet das Verhältnis zwischen Vorsteher und Stiftung ein Passus im 1431 aufgesetzten Testament Johann Reses, des Vorgängers Hinrich Langes als Provisor des Nikolaihofes. Rese ließ dort festhalten, dass sich in seinem Besitz ein Rentenbrief des Lüneburger Rates über 36 Mark befinde, der auf ihn und seine Erben ausgeschrieben sei. Diese Rente aber - so heißt es weiter - gehöre dem Nikolaihof und sei mit dessen Geld erworben worden. ${ }^{34}$ Eine ähnliche Vermögens- und Abrechnungspraxis kennen wir auch aus anderen Bereichen administrativen Handelns im Mittelalter.

\footnotetext{
${ }^{30}$ Irene STAHL, Lüneburger Ratslinie 1290-1605, in: NdSächsJb. 59, 1987, S. 139-187, hier S. 167.

${ }^{31}$ Stadtarchiv Lüneburg, Amtsbücher (im Folgenden: StAL, AB) 362.

${ }^{32}$ StAL, AB 362, S. 157.

${ }^{33}$ StAL, AB 383", fol. 45r-46v.

${ }^{34}$ Uta REINHARIT (Bearb.), Lüneburger Testamente des Mittelalters. 1323 bis 1500 (Veröffentlichungen der Historischen Kommission für Niedersachsen und Bremen 37), Hannover 1996, Nr. 165 (S. 227f.).
} 
IV.

Das Projekt verankert die Sozialgeschichte der .Armenfürsorge" im sozialen Raum der Stadt. Das damit bezeichnete komplexe Beziehungsfeld ist durch mehrere Elemente bestimmt: In den Blick zu nehmen sind insbcsondere Familien, (korporative) Gruppen, Rat und Gemeinde, Stadtherren und Kirche. Es geht um soziale Gruppen und Netzwerke, um Kooperation und Konkurrenz, um genossenschaftliche Strukturen und die Konfrontation mit einer sich langsam herausbildenden ,obrigkeitlichen ' Politik des Rates. ${ }^{35} \mathrm{Da}$ sich der soziale Raum im realen städtischen Raum spiegelt, werden auch topographische Fragen, Hospitalbauten und andere Formen der Repräsentation im ,öffentlichen ' oder ,teilöffentlichen' Raum einbezogen. ${ }^{36}$ Und schließlich sind Aspekte der Regionalität zu beachten, wenn man so will also der Verknüpfung verschiedener städtischer Räume. Um wenigstens den letzten Punkt kurz zu konkretisieren, sei auf Fälle verwiesen, in denen karitative Stiftungen an auswärtiger Stelle errichtet wurden. So nahm z. B. der Lübecker Goldschmied Hans Bruns Mitte des 15. Jahrhunderts quasi eine Neustiftung des Heilig-Geist-Hospitals in der Kleinstadt Oldesloe vor. ${ }^{37}$ Der Ort stand ökonomisch unter starkem lübeckischen Einfluss, und auch Hans Bruns war dort durch seine Beteiligung an

35 Jörg RogGE, Für den Gemeinen Nutzen. Politisches Handeln und Politikverständnis von Rat und Bürgerschaft in Augsburg im Spätmittelalter (Studia Augustana 6), Tübingen 1996; Olaf MÖRKE. Daseinsvorsorge in Städten der niederländischen Republik. Bemerkungen zur Persistenz des alteuropäischen Gemeindekorporatismus, in: Städtisches Gesundheits- und Fürsorgewesen (wie Anm. 8), S. 125-150.

${ }^{36}$ Zur städtischen Topographie: Die Sozialstruktur und Sozialtopographie vorindustrieller Städte. Beiträge eines Workshops am Institut für Geschichte der Martin-Luther-Universität Halle-Wittenberg am 27. und 28. Januar 2000, hg. von Matthias MEINHARDT und Andreas RANFT (Hallische Beiträge zur Geschichte des Mittelalters und der Frühen Neuzeit 1), Berlin 2005; Karsten IGEL, Der Raum als soziale Kategorie. Methoden sozialräumlicher Forschung am Beispiel des spätmittelalterlichen Greifswalds, in: Städtesystem und Urbanisierung im Ostseeraum in der Frühen Neuzeit. Urbane Lebensräume und Historische Informationssysteme. Beiträge des wissenschaftlichen Kolloquiums in Rostock vom 15. und 16. November 2004, hg. von Stefan KROLL und Kersten KRÜGER (Geschichte: Forschung und Wissenschaft 12), Berlin 2006, S. 265-300. - Zur Repräsentation im Zusammenhang mit Stiftungen und Hospitälern: Ilka S. MINNEKER, Repräsentation und sakrale Legitimation. Majestas Domini und Bürgermedaillons im Heilig-Geist-Hospital zu Lübeck, in: ZVLGA 79, 1999, S. 24-74: Dietrich W. POECK, Wohltat und Legitimation, in: Städtisches Gesundheits- und Fürsorgewesen (wie Anm. 8), S. 1-17; Stefanie RÜTHER, Prestige und Herrschaft. Zur Repräsentation der Lübecker Ratsherren in Mittelatter und Früher Neuzeit (Norm und Struktur 16), Köln 2003. - Zur städtischen ,Öffentlichkeit‘: Andrea LöTHER, Städtische Prozessionen zwischen repräsentativer Öffentlichkeit, Teilhabe und Publikum, in: Das Öffentliche und Private in der Vormoderne, hg. von Gert MELviLlE und Peter von Moos (Norm und Struktur 10), Köln 1998, S. 435-459.

\footnotetext{
${ }^{37}$ MeINerT, Hospitäler (wie Anm. 13), S. 79.
} 
der Oldesloer Saline wirtschaftlich engagiert. ${ }^{38}$ Abstrakt ließe sich von der Präsenz im fremden Raum mittels karitativer Stiftungen sprechen. Und Hans Bruns war keineswegs der einzige Lübecker, der auf diese Weise aktiv wurde: So bedachte der Domdekan Johannes Brandes $(\dagger 1531)$ bei einer Stipendien- und Aussteuerstiftung ausdrücklich auch seine Heimatstadt Itzehoe, ${ }^{39}$ und der Vikar Nikolaus Petri setzte 1553 in Husum eine jährlich Rente von insgesamt 30 Mark zur genau beschriebenen Verteilung an Arme aus. ${ }^{40}$

Auch Hans Berskamp und Hermann Evinghusen wollten sich nicht mit der Stiftung ihres Lübecker Armenganges begnügen. Nach dem eingangs zitierten Testament Hans Berskamps von 1473 planten beide, auch in Wismar gemeinsam ein Armenhaus zu errichten, und zwar nach dem Muster ihrer Lübecker Stiftung, d. h. zum Beispiel unter Vermietung einiger zugehöriger Buden und Keller zur Erwirtschaftung notwendiger Einnahmen. Berskamp traf auch gleich Bestimmungen hinsichtlich der späteren Verwaltung dieser zweiten Stiftung. Charakteristischerweise wurden in diesem Fall - anders als für den Armengang in der Lübecker Schlumacherstraße - keine individuellen Vorsteher eingesetzt - die oft dahinterstehenden verwandtschaftlichen Netzwerke könnten den beiden Lübeckern in Wismar gefehlt haben. Statt dessen sollte das künftige Armenhaus einer Wismarer gilde anvertraut und damit auf andere Weise im sozialen Raum der Stadt verankert werden.

\footnotetext{
${ }^{38}$ Friedrich BANGERT, Geschichte der Stadt und des Kirchspiels Oldesloc, Bad Oldesloe 1925, S. 58-68 und passim sowie S. 83 (zu Hans Bruns).

${ }^{39}$ UBBL IV, 2361. Zu Brandes s. Wolfgang PRANGE, Vikarien und Vikare in Lübeck bis zur Reformation (Veröffentlichungen zur Geschichte der Hansestadt Lübeck B 40), Lübeck 2003, S. 137.

${ }^{40}$ Husumer Urkundenbuch 1429-1609, bearb. von Ernst MölLER (Schriften des NissenHauses, Husum 1), Husum 1939, Nr. 463.
} 